\section{Index of Countries}

Albania 26,

Algeria 26, 171ff, 227, 230,

Angola 101,

Argentina $26,65,66,72,75,77,115$,

313.

Armenia 26, 115, 354-5.

Australia 20, 26, 102, 106, 322 .

Austria 26, 97, 98, 115,

Azerbaijan 26, 167,

Bahrain 26,

Belgium 23, 26, 97, 98, 115,

Bolivia 26, 65, 230,

Botswana 101,

Brazil 26, 65, 66, 72, 75, 77, 98, 102, 115,340 .

Brunei 200.

Bulgaria 26, 102, 115, 230, 340.

Burma (Myanmar) 313, 350.

Cambodia 350.

Canada 4ff, 19, 26, 115, 126, 253.

Chile 9, 18, 26, 33, 98, 115, 272, 313.

China (Beijing) 9, 26, 80ff, 115, 133, 210ff, 245, 319ff, 355-6.

(Taipei) 23, 26, 115, 163, 219, 320ff.

Colombia 26, 115.

Costa Rica 70, 72, 78 .

Croatia 26, 115.

Cuba 23, 26, 115, 163.

Czech Republic 340.

Denmark 26, 97, 98, 115.

Ecuador 26, 233.

Egypt 26, 157ff, 167, 179ff.

El Salvador 70.

Estonia 23, 25, 26, 97, 115.

Finland 26, 97, 98, 115.

France 26, 45, 56, 59, 72, 97, 98, 133, 176, 178, 276-7, 354-5.
Georgia (Rep. of) 26, 97, 98 .

Germany 26, 97, 98, 115, 181, 222, 272, 354-5.

Greece 26, 97, 98, 115, 367.

Honduras 26, 69ff.

Hungary 26, 102, 115.

Iceland 23 .

India $3,9,14,26,86,115,133,164$, $165,258,272$, 303ff, 313, 322, 326, 340, 354-5, 369 .

Indonesia 9, 26, 115, 133, 197ff, 233, 313.

Iran 26, 115, 188.

Iraq 26, 157.

Ireland 26, 340 .

Israel 26.

Italy $26,61,97,98,115,354-5$.

Japan $11,18,21,25,26,47,56 \mathrm{ff}, 67$, $115,166,184,250,266,271,312-3,319 \mathrm{ff}$, 354-5.

Jordan 160, 163, 313.

Kazakhstan 26.

Korea (Dem. Rep.) 23, 26.

Korea (Rep. of) 9, 21, 27, 115, 133, $319 f f$.

Laos 350.

Latvia 26, 97, 98.

Lithuania 26, 115, 340.

Luxembourg 97, 98.

Macedonia (Rep. of) 232.

Madagascar 101.

Malaysia 26, 200, 313.

Malta 340.

Mauritius 26, 227.

México 26, 66, 107ff, 115, 164, 165, 167, 178, 208.

Morocco 26, 59ff, 178, 227.

Mozanbique 101.

Netherlands 26, 39, 97, 98, 115, 126, $257,265$. 
New Zealand 9, 11, 18ff, 26, 115, 116, Vatican City State 8, 23, $110 \mathrm{ff}$. $126,130,163,208,222,269-70,322$. Venezuela $27,114,115,205 f f$.

Nigeria 19, 26, 115, 227, 370.

Norway 23, 26, 97. Vietnam 27, 46ff, 92, 115, 262ff, 313, 348,350 .

Pakistan 26.

Paraguay 27, 65ff, 313, 315 .

Peru 27, 115, 368-9.

Yugoslavia 23, 27.

Philippines 27, 49ff, 313.

Poland 27, 115, 222.

Zambia 39ff, 101.

Zimbabwe 101, 364.

Portugal 27, 64, 97, 115.

Romania 27, 102, 115, 164, 232.

Russia 27, 97, 98, 115, 167, 188, 230, 355-6.

Saudi Arabia 23, 160.

Singapore 21, 27, 271, 313.

Slovakia 27, 340 .

Slovenia 27, 115.

Somalia 191.

South Africa 27, 86, 101, 115, 117ff, $133,152,221 \mathrm{ff}, 348-9$.

Spain 27, 64, 72, 78, 97, 98, 115, 133, 159, 166, 176, 277, 337, 354-5.

Sri Lanka 27, 115, 266ff, 279-80, 285ff, 313.

Sweden 27, 97, 98, 115, 191.

Switzerland 23, 27, 97, 115.

Tajikistan 27, $187 \mathrm{ff}$.

Tanzania 166, 340 .

Thailand 27, 56, 133, 165, 243ff, 313, 350.

Turkey 27, 133, 188.

Ukraine 25, 27, 115.

United Kingdom 20, 27, 64, 97, 106, $115,131 \mathrm{ff}, 176,179,180,222,338,334-$ 5.

United States of America 20ff, 27, 48, $54,85,110,115,139,142,152,222-3$, $271,276,285,287,313,326,338,355-$ 6,372 .

Uruguay $27,65,115,313$.

Uzbekistan 27, 166, 230, 232, 313. 